\title{
Omics-based Medicine and Systems Pathology A New Perspective for Personalized and Predictive Medicine
}

\author{
H. Tanaka ${ }^{1}$ \\ ${ }^{1}$ Graduate School of Biomedical Science, Tokyo Medical and Dental University, Tokyo, Japan
}

\begin{abstract}
Summary
Objectives

Recent important advances in the human genomics and post-genomic "omics" are now bringing about a new medical care which we call "omics-based medicine". In this article, we investigated the development and future possibilities of omics-based medicine.
\end{abstract}

\section{Methods}

We divided the development of omicsbased medicine into three generations in order to clarify the main clinical goals and characteristics of informatics method of each generation, together with its future possibilities.

\section{Results}

The first generation of omics-based medicine started with "genomic medicine" based on the inborn individual differences of genome. It has opened the study of genetic polymorphism of the diseases and promoted the personalized medication based on the pharmacogenetic /pharmacogenomic difference of the drug response. In the second generation of omics-based medicine, owing to the advances in the high-throughput technology, vast amount of the various post-genomic disease omics data containing comprehensive molecular information of diseased somatic cells has become available. It reflects the ongoing state of diseases more closely and enables the predictive medicine such as prognosis prediction of disease by applying the datadriven analysis. Finally, due to the rapidly growing knowledge about the cellular molecular network, system-level understanding of the disease, called systems pathology, becomes possible. It can fully exploit the substantial contents of disease omics and will lead to a comprehensive understanding of disease process by using model-driven analysis.
Conclusion: Omics-based medicine and systems pathology will realize a new personalized and predictive medicine.

\section{Keywords}

Genomics, pharmacogenomics, systems pathology, Omics-based medicine, omics

DOI

10.3414/ME9307

The full English version can be found in Methods of Information in Medicine 2010 49 2: $173-185 /$ or

http://www.schattauer.de/de/magazine/ue bersicht/zeitschriften-az/methods/issue/special/manuscript/1275 7/show.html. 